accounted for $8.5 \%$ of childhood brain tumors, while other series report a frequency of less than $5 \%$. Germinomas arising in the pineal or suprasellar region are the most common form (65\%) of PGCT and have a good prognosis, with $88 \%$ long-term survival after surgical removal and radiation. Severe residual complications, including cognitive deficits, are uncommon, despite post-operative craniospinal axis irradiation. Malignant teratomas have a less favorable prognosis. The younger age at which medulloblastomas are treated with irradiation accounts for the higher incidence of cognitive deficits in these tumor patients.

\title{
HYPOXIC SYNDROMES
}

\section{BREATH-HOLDING SPELLS}

The clinical characteristics, types, diagnosis, and management of breath-holding spells are reviewed from The Department of Pediatrics (Neurology), Park Nicollet Medical Center, Minneapolis, MN. There are two forms, pallid and cyanotic. Pallid breath-holding spells (BHS) result from vagal hyperresponsiveness, following a sudden, unexpected, unpleasant stimulus, usually a mild head injury. Cardiac monitoring reveals prolonged asystoles, which can also be induced by ocular compression, and is accompanied by syncope or an anoxic seizure. Vagal cardiac inhibition with cerebral anoxia is the pathophysiology of pallid BHS. Cyanotic breath-holding spells result from a complex interplay of hyperventilation followed by apnea in expiration, and increased intrathoracic pressure. Whereas pallid BHS occur after injury, cyanotic BHS are precipitated by anger. Diagnosis may be confirmed by EEG with ocular compression and cardiac monitoring. A pallid spell is associated with cardiac asystole and EEG hypersynchronous slowing. Cyanotic spells have similar EEG changes without bradycardia or asystole. Prolonged QT syndrome is a rare but serious cause of anoxic seizure, induced by exercise, injury, or fright. Cerebral hypoxia may result from ventricular tachycardia. More protracted loss of consciousness with hypotension may indicate a cardiac pathology. A more protracted seizure following a BHS may represent an anoxic-epilepsy, requiring anticonvulsant therapy. Spontaneous remission of BHSs is to be expected, but parents require frequent reassurance about the benign nature of the spells. (Breningstall GN. Breath-holding spells. Pediatr Neurol Feb 1996;14:91-97). (Respond: Dr Breningstall, Pediatric Subspecialties, Park Nicollet Medical Center, 910 East 26th St, Suite 325, Minneapolis, MN 55404).

COMMENT. Although anticonvulsant therapy is sometimes advisable when the convulsive episode is prolonged and represents an anoxic-epilepsy, traditional therapy will not generally prevent the breath-holding spell. Perhaps some of the newer antiepileptic medications should be tried in children with numerous attacks. The therapeutic nihilistic approach to BHS practiced by many physicians is often difficult for a parent to accept. An iron deficiency anemia may be an underlying causative factor in about 20\% of cases of breath-holding (Holowach J, Thurston DL. N Eng I Med 1963;268:21). Neurologic deficits with iron deficiency anemia are discussed in Progress in Pediatric Neurology 1991, PNB Publ, pp397-8.

Apnea and bradycardia during epileptic seizures were studied at the Telemetry Unit, National Hospital for Neurology and Neurosurgery, Queen Square, London. (Nashef L et al. I Neurol Neurosurg Psychiatry March 
1996;60:297-300). Apnea occurred in 20 of 47 clinical seizures and 10 of 17 patients. It was generally central, but obstructive apnea occurred in 3 . Oxyhemoglobin saturation dropped to $<85 \%$, and tachycardia was common. Bradycardia/sinus arrest was documented with a change in respiration in 4 . Similar mechanisms involving cardiorespiratory reflexes are suggested in relation to cases of sudden death in epilepsy.

\section{DIABETES INSIPIDUS WITH HYPOXIC ENCEPHALOPATHY}

Neurogenic diabetes insipidus (DI) due to hypoxic encephalopathy is reported in 6 cases and 28 pediatric cases in the literature are reviewed from the Mackay Memorial Hospital, Taipei, Taiwan, ROC. The ages ranged from infancy to 18 years (mean 7 years), and the interval from the hypoxia to the onset of DI was a mean of 4 days. Two neonates survived but had developmental delay. The remaining 26 patients died. DI is a sign of severe brain damage, and this potential complication should be suspected in patients with polyuria and dehydration following hypoxic encephalopathy. (Lee Y-J, Huang F-Y et al. Neurogenic diabetes insipidus in children with hypoxic encephalopathy: six new cases and a review of the literature. Eur I Pediatr March 1996;155:245-248). (Respond: Dr Fu-Yuan Huang, Department of Paediatrics, Mackay Memorial Hospital, Taipei, 10449 Taiwan, ROC).

COMMENT. Neurogenic diabetes insipidus may be caused by head trauma, meningitis, encephalitis, a complication of brain surgery, in addition to hypoxia. Fluid intake and output, plasma sodium, and urine specific gravity should be monitored in children following hypoxic/ischemic insults or in other critically ill brain injured patients.

\section{INFECTIOUS DISEASES}

\section{NEUROLOGIC SIGNS OF LYME DISEASE}

Clinical manifestations of Lyme disease (LD) in 97 seropositive children were reviewed at the Children's Hospital of Philadelphia, with particular attention to neurologic symptoms and signs. Of 69 children with LD, 22 (32\%) had new neurologic abnormalities, mainly facial palsy and aseptic meningitis. Only $27 \%$ of children with neurologic abnormalities due to LD had a history of erythema migrans or arthritis. Seropositivity for LD and neurologic symptoms usually indicates an active neuroborreliosis. (Bingham PM et al. Neurologic manifestations in children with Lyme disease. Pediatrics Dec 1995;96:10531056). (Respond: Dr Peter M Bingham, Division of Neurology, Children's Hospital of Philadelphia, 34th Street and Civic Center Blvd, Philadelphia, PA 19104).

COMMENT. Definite evidence for neuroborreliosis requires characteristic neurologic abnormalities and either erythema migrans, arthritis or heart involvement, positive spinal fluid serology, or seroconversion. Peripheral neuropathy is infrequent in children compared to adults. Facial palsy and aseptic meningitis are the most frequent neurologic manifestations of $\mathrm{LD}$ in children. In the approaching summer months, children presenting with these disorders should be checked for possible Lyme disease. 